\title{
Sexual Function in Men with Castrate Levels of Testosterone: Observations of a Subgroup of Sexually Active Men with Prostate Cancer Undergoing Androgen Deprivation Therapy
}

\author{
Evan $\mathrm{Ng}^{*}$, Tammy Corica ${ }^{2}$, Sandra Turner ${ }^{3}$, Adeline Lim $^{4}$, Nigel Spry ${ }^{2}$ \\ ${ }^{1}$ Genesis CancerCare WA, Perth, WA, Australia \\ ${ }^{2}$ Department of Radiation Oncology, Sir Charles Gairdner Hospital, Nedlands, WA, Australia \\ ${ }^{3}$ Crown Princess Mary Cancer Centre, Westmead Hospital, Sydney, NSW, Australia \\ ${ }^{4}$ Department of Radiation Oncology, Austin Health, Melbourne, VIC, Australia \\ Email: *evanng8@gmail.com
}

Received 18 May 2014; revised 10 June 2014; accepted 18 July 2014

Copyright (C) 2014 by authors and Scientific Research Publishing Inc.

This work is licensed under the Creative Commons Attribution International License (CC BY). http://creativecommons.org/licenses/by/4.0/

(c) (i) Open Access

\section{Abstract}

Purpose: To identify possible factors that influence sexual function in men undergoing maximal androgen deprivation therapy (ADT). Patients and Methods: A descriptive exploration was performed looking at characteristics of twenty-two men reporting sexual activity after nine months of maximal ADT. This previously published Phase II study, involved 250 prostate cancer patients undergoing intermittent ADT. An analysis between this cohort and the group that did not maintain sexual function was performed to ascertain if age, testosterone level, functional status or maintenance of quadriceps strength had an impact upon sexual function. Results: There was no difference in age, testosterone level or ECOG performance status between the sexually active and nonsexually active groups. Over the course of 9 months of ADT, the sexually active group appeared to maintain quadriceps muscle strength as measured with physical stands, and maintained overall health as measured by quality of life questionaries, compared to the non-sexually active group. Conclusions: This retrospective study suggests that exercise during ADT may reduce the impact of ADT on sexual function. This warrants further testing, and could be the focus of future randomised controlled trials.

\section{Keywords}

Prostate Cancer, Intermittent Androgen Suppression, Exercise, Sexual Function,

\footnotetext{
${ }^{*}$ Corresponding author.
}

How to cite this paper: Ng, E., Corica, T., Turner, S., Lim, A. and Spry, N. (2014) Sexual Function in Men with Castrate Levels of Testosterone: Observations of a Subgroup of Sexually Active Men with Prostate Cancer Undergoing Androgen Deprivation Therapy. Open Journal of Urology, 4, 98-103. http://dx.doi.org/10.4236/oju.2014.47017 


\section{Leuprolide, Flutamide}

\section{Introduction}

Prostate cancer is the second most common male cancer worldwide [1]. More than 2000 men in Australia commence androgen deprivation therapy (ADT) each year as part of their treatment for prostate cancer [2].

We have previously published data on the influence of testosterone suppression on sexual function on 250 men with prostate cancer, undergoing intermittent ADT [3]. These data found a subgroup of men, who in spite of ADT which lead to castrate levels of testosterone, reported maintenance of sexual activity. In this report, we aim to identify factors that may predict for, or result in, maintenance of sexual function by comparing the characteristics of patients in this subgroup with the cohort who did not maintain sexual function.

\section{Methods}

\subsection{Eligibility and Treatment Program}

This multicentre study began in July 1999, registered 250 men across Australia and closed to follow up in July 2004.

Study participants were required to have a histological or cytological diagnosis of adenocarcinoma of the prostate and Eastern Cooperative Oncology Group (ECOG) performance status of 0 - 2. Full details of this cohort are described in an earlier publication [4]. Androgen suppression therapy (ADT) was achieved by maximal androgen deprivation using Flutamide (Eulexin $\left.{ }^{\circledR}\right) 250$ mg tds and Leuprolide (Lucrin $\left.{ }^{\circledR}\right) 22.5$ mg three monthly depot, and continued for a scheduled nine months (ONPhase), at which point patients ceased therapy providing their PSA level was equal to or below $4 \mathrm{ng} / \mathrm{mL}$ (OFFPhase). Assessments were undertaken at baseline, and three monthly intervals throughout the study period.

\subsection{Sexual Function and Quality of Life Questionnaire}

Participants completed the European Organisation for Research and Treatment of Cancer (EORTC) QLQ-C30 version 2.0 core quality of life (QOL) questionnaire and prostate specific module QLQ-PR25 version 3.0, results as previously described [3]. Questions used to assess sexual function assessment are presented in Table 1, where scores were graded on a Likert scale. Questions used to assess general health are presented in Table 2.

Table 1. Questions pertaining to sexual function.

\begin{tabular}{llccc}
\hline & During the last 4 weeks, & Not at all & A little & Quite a bit Very much \\
\hline Q49 & Have you felt less masculine as a result of your illness or treatment? (Masculinity) & 1 & 2 & 3 \\
Q50 & To what extent were you interested in sex? (Libido) & 1 & 2 \\
Q51 & To what extent were you sexually active (with or without intercourse)? (Activity) & 1 & 2 \\
& Please answer the next question only if you have been sexually active over last 4 weeks, & 4 \\
Q53 & Did you have difficulty getting or maintaining an erection? (Potency) & 1 & 2 \\
\hline
\end{tabular}

Table 2. Questions pertaining to general health.

Q1 Do you have any trouble doing strenuous activities like carrying a heavy shopping bag or suitcase?
Q2
Fo you have any trouble taking a long walk?
F29
Very poor


Our previous publication [3] identified a subgroup of patients who were sexually active after 9 months of ADT. This paper explores the longitudinal data set associated with this subgroup and contrasts the characteristics of these men with the non sexually active cohort. The responses to the activity question "To what extent were you sexually active (with or without intercourse)" were examined. Excluding patients not sexually active at baseline, after 9 months of ADT, 22 men responded being sexually active (sexually active group). Characteristics of these men were compared to the remaining cohort (non-sexually active group) using the following measures.

\subsection{Age and Testosterone Levels}

Patient age was recorded at study entry. Serum testosterone was measured at baseline, at the three month mark of ONPhase, and three monthly during OFFPhase, until testosterone levels returned to baseline.

\subsection{Quality of Life Questionnaire}

Questions 1, 2 and 29 were selected as measures to assess general health.

\subsection{Quadriceps Strength Measurement}

The time required for patients to complete 20 full stands from a sitting position was measured. This was done in a separate part of the trial to measure quality of life and long term physiological changes associated with ADT. Use of the arms to assist standing was not allowed. Participants were encouraged to perform the task as quickly as possible. The time taken was measured in seconds (s). Measurement of the time to complete 10 stands was also recorded.

\section{Statistical Analysis}

Characteristics of the subgroup of 22 men, who were sexually active after 9 months of ADT were compared to the remaining cohort.

For continuous variables we carried out two sample t-tests, whilst for categorical variables, chi-squared tests were used. P-values are presented in Table 3 and Table 4.

\section{Results}

Between 1999 and 2004, 250 men were recruited to the study investigating the effects of intermittent ADT. They all underwent maximal ADT, and have been analysed as two groups: sexually active and non-sexually active, as described above. Castrate level of testosterone was confirmed at 3 months in all patients, which was defined as less than $2 \mathrm{nmol} / \mathrm{L}$.

Age, testosterone levels, ECOG Performance Status, responses to general health assessment and quadriceps strength measurement were compared for the two groups.

There was no statistically significant impact demonstrated for age, baseline testosterone levels and baseline ECOG performance status, on sexual activity at 9 months as seen in Table 3.

\subsection{General Heath Assessment}

For all three general health questions analysed, there was no significant difference between the sexually active group and non-sexually active group at baseline. The sexually active group maintained better general health scores for all three questions after 9 months of ADT, as detailed in Table 4.

Between baseline and the 9-month time point, the sexually active group demonstrated no significant change in their responses with regards to the ability to perform strenuous activities (Q1). Conversely more patients in the non-sexually active Group reported trouble with strenuous activities at 9 months $(\mathrm{p}<0.01)$.

Similarly, the sexually active group reported no significant change in terms of trouble taking a long walk (Q2) between baseline and 9 months, whereas the sexually inactive group reported a significant change in this ability during the same time period $(\mathrm{p}<0.01)$.

Furthermore, with respect to overall heath (Q29), the sexually active group had no significant change in their self-rated scores between baseline and 9 months, while a significantly lower self-assessment of overall health 
Table 3. Physical characteristics between sexually active group versus non-sexually active group after 9 months of ADT.

\begin{tabular}{|c|c|c|c|}
\hline & Sexually Active & Non-Sexually Active & $P$ values \\
\hline Age (years) & 69.32 & 69.95 & 0.7460 \\
\hline \multicolumn{4}{|c|}{ ECOG Status (within the group, \% of men with ECOG 0) } \\
\hline At Baseline & 73 & 75 & 0.7804 \\
\hline After 9 months of ADT & 59 & 68 & 0.7434 \\
\hline \multicolumn{4}{|c|}{ Testosterone Levels (nmol/L) } \\
\hline At Baseline & 16.12 & 14.86 & 0.3290 \\
\hline
\end{tabular}

Table 4. General health assessment.

\begin{tabular}{|c|c|c|c|}
\hline \multicolumn{4}{|l|}{ General Heath Questionnaire } \\
\hline & At Baseline & After 9 months of ADT & \\
\hline \multicolumn{4}{|l|}{ Q1 (\% responded Y) } \\
\hline Sexually Active & 14 & 19 & 0.9398 \\
\hline Non-Sexually Active & 15 & 36 & 0.0021 \\
\hline \multicolumn{4}{|l|}{ Q2 (\% responded Y) } \\
\hline Sexually Active & 18 & 33 & 0.1573 \\
\hline Non-Sexually Active & 24 & 44 & 0.0003 \\
\hline \multicolumn{4}{|l|}{ Q29 } \\
\hline Sexually Active & 5.95 & 5.32 & 0.0129 \\
\hline Non-Sexually Active & 5.53 & 5.10 & 0.0020 \\
\hline \multicolumn{4}{|c|}{ Quadriceps Strength Measurement (sec) } \\
\hline Sexually Active & 20.77 & 16.87 & 0.0007 \\
\hline Non-Sexually Active & 22.38 & 20.93 & 0.0371 \\
\hline
\end{tabular}

was noted in the non-sexually active group, after 9 months of ADT (p < 0.01).

\subsection{Quadriceps Strength Measurement}

There was a significant difference in quadriceps strength measurement after 9 months of ADT between the sexually active and non-sexually active groups. At baseline, mean time taken to complete 10 stands was similar between the sexually active group and non-sexually active group; $20.77 \mathrm{~s}$ and $22.38 \mathrm{~s}$ respectively.

After 9 months of ADT, mean time taken to complete 10 stands, when comparing groups, was $16.87 \mathrm{~s}$ in the sexually active group, versus $20.93 \mathrm{~s}$ in the non-sexually active group $(\mathrm{p}<0.01)$. Noting the time taken for the sexually active men decreased, suggesting improved quadriceps strength.

\section{Discussion}

Results from this subgroup analysis suggest maintaining muscle strength during ADT may in some way counteract the adverse effect of ADT on sexual function.

In a previous publication data were presented where at baseline, prior to administration of ADT, participants had a median age of 71 and $46 \%$ were still sexually active [3]. These men underwent maximal ADT, and despite castrate levels of testosterone, a subgroup of 22 men remained sexually active. These 22 men represented $20 \%$ of patients who were sexually active at baseline.

There are several known facts about sexual activity. Firstly, that sexual activity decreases with age [5]. Secondly, sexual activity decreases with decreasing levels of testosterone [3]. The men in this trial, who underwent ADT, all reached castrate levels of testosterone. Hence there was some speculation that the subgroup of sexually active men on ADT were perhaps younger, or had a slightly higher levels of testosterone. Analysis of this subgroup has shown this was not the case. 
Quadriceps muscle strength, which was measured as part of the trial to document muscle loss over the course of ADT, turned out to be significant between the sexually active and non-sexually active men. At baseline, quadriceps muscle strength, measured as timed stands, was similar between the two groups. However after 9 months of ADT, the sexually active group appeared to have superior quadriceps muscle strength, compared to the non-sexually active group. Of note, in both groups the time it took to complete 10 stands was shorter after 9 months post commencement of ADT compared to baseline (pre-ADT). The drop in the non-sexually active group was not statistically significant. The decrease in time in both groups may be attributable to patients having become familiar with the timed stands task, whilst the significant reduction in time in the sexually active group may reflect an increase in muscle mass, potentially due to exercise.

Exercise has shown benefits in multiple trials, ranging from reduction in cardiovascular disease and type II diabetes mellitus [6] [7]. In the setting of ADT, exercise has been shown to be effective in reducing muscle and bone loss as well as combating fatigue. A recent randomized controlled trial involving 57 patients has also reported that short-term exercise during ADT results in maintenance of sexual function in the exercise group [8]. The results of this current analysis raise the interesting hypothesis that the men who exercise and maintain their muscle mass over the course of ADT have a higher probability of maintaining sexual function.

The subgroup of sexually active men showed improved quadriceps strength, when compared to the nonsexually active men. One possibility is that fitter men were more likely to maintain sexual function, but this seems less likely given baseline measurements, and general health assessment questions, in this trial failed to detect any difference in the two groups. A more likely possibility is that the men, who exercised and maintained their muscle mass over the course of ADT, had a higher probability of maintaining sexual function. This is as demonstrated by quadriceps strength assessment and general health assessment results.

There are several limitations of this study. These include the retrospective nature of the data, and the small number of patients in the sexually active subgroup. Assessment of general health was subjective in nature, with the answering of questionnaires. Despite a formal protocol being in place, the measurement of quadriceps muscle strength may not have provided highly reliable data, given that different clinic nurses at multiple participating sites collected the measurements. Although an interesting hypothesis, validation of this concept requires further investigation, with more sophisticated and rigorous testing.

\section{Conclusion}

This study generates the hypothesis that exercise during ADT may impact favourably on the side effect profile of ADT from the perspective of sexual function. This warrants further testing, and could be the focus of future randomised controlled trials.

\section{Funding}

Supported by Abbott Australasia Pty Ltd and Schering-Plough Pty Ltd.

\section{References}

[1] Jemal, A., Murray, T., Samuels, A., et al. (2003) Cancer Statistics, 2003. CA: A Cancer Journal for Clinicians, 53, 526. http://dx.doi.org/10.3322/canjclin.53.1.5

[2] AIHW (Australian Institute of Health and Welfare) \& ACCR (Australasia Association of Cancer Registries) (2007) Cancer in Australia: An Overview, 2006. Cancer Series No. 37, Cat No. CAN 32, AIHW, Canberra.

[3] Ng, E., Woo, H., Turner, S., et al. (2012) The Influence of Testosterone Suppression and Recovery on Sexual Function in Men with Prostate Cancer: Observations from a Prospective Study in Men Undergoing Intermittent Androgen Suppression. The Journal of Urology, 187, 2162-2167. http://dx.doi.org/10.1016/j.juro.2012.01.080

[4] Spry, N., Kristjanson, L., Hooton, B., et al. (2006) Adverse Effects to Quality of Life Arising from Treatment Can Recover with Intermittent Androgen Suppression in Men with Prostate Cancer. European Journal of Cancer, 42, 10831092. http://dx.doi.org/10.1016/j.ejca.2006.01.029

[5] Davidson, J.M., Chen, J.J., Crapo, L., et al. (1983) Hormonal Changes and Sexual Function in Aging Men. The Journal of Clinical Endocrinology \& Metabolism, 57, 71-77. http://dx.doi.org/10.1210/jcem-57-1-71

[6] Blair, S.N., Kampert, J.B., Kohl III, H.W., et al. (1996) Influences of Cardiorespiratory Fitness and Other Precursors on Cardiovascular Disease and All-Cause Mortality in Men and Women. JAMA, 276, 205-210. http://dx.doi.org/10.1001/jama.1996.03540030039029 
[7] Helmrich, S.P., et al. (1991) Physical Activity and Reduced Occurrence of Non-Insulin-Dependent Diabetes Mellitus. The New England Journal of Medicine, 325, 147-152. http://dx.doi.org/10.1056/NEJM199107183250302

[8] Cormie, P., Newton, R.U., Taaffe, D.R., et al. (2013) Exercise Maintains Sexual Activity in Men Undergoing Androgen Suppression for Prostate Cancer: A Randomized Controlled Trial. Prostate Cancer and Prostatic Disease, 16, 170175. 
Scientific Research Publishing (SCIRP) is one of the largest Open Access journal publishers. It is currently publishing more than 200 open access, online, peer-reviewed journals covering a wide range of academic disciplines. SCIRP serves the worldwide academic communities and contributes to the progress and application of science with its publication.

Other selected journals from SCIRP are listed as below. Submit your manuscript to us via either submit@scirp.org or Online Submission Portal.
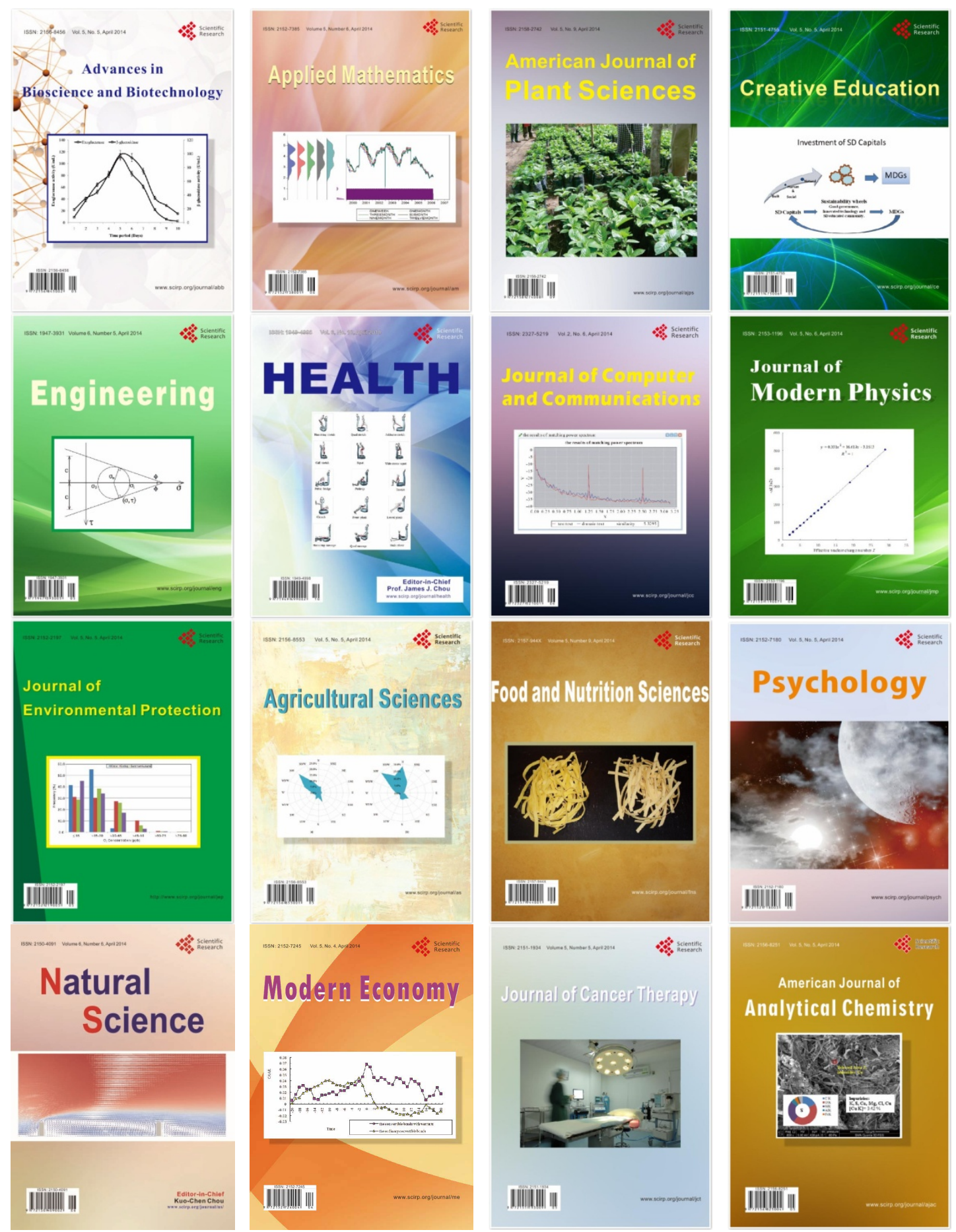